\title{
A Determinant Identity that Implies Rogers-Ramanujan
}

\author{
Kristina C. Garrett \\ Department of Mathematics and Computer Science \\ Carleton College, Minnesota, USA \\ kgarrett@carleton.edu
}

Submitted: Oct 2, 2004; Accepted: Nov 23, 2004; Published: Jul 29, 2005

MR Subject Classifications: 05A30, 33C45

\begin{abstract}
We give a combinatorial proof of a general determinant identity for associated polynomials. This determinant identity, Theorem 2.2 , gives rise to new polynomial generalizations of known Rogers-Ramanujan type identities. Several examples of new Rogers-Ramanujan type identities are given.
\end{abstract}

\section{Introduction}

The Rogers-Ramanujan identities are well known in the theory of partitions. They may be stated analytically as

$$
\begin{aligned}
& \sum_{n=0}^{\infty} \frac{q^{n^{2}}}{(q ; q)_{n}}=\frac{1}{\left(q, q^{4} ; q^{5}\right)_{\infty}} \\
& \sum_{n=0}^{\infty} \frac{q^{n^{2}+n}}{(q ; q)_{n}}=\frac{1}{\left(q^{2}, q^{3} ; q^{5}\right)_{\infty}}
\end{aligned}
$$

where

$$
(a ; q)_{n}=(1-a)(1-a q) \cdots\left(1-a q^{n-1}\right) \quad \text { for } \quad n \geq 0, \quad(a ; q)_{0}=1
$$

and

$$
(a ; q)_{\infty}=\prod_{n=0}^{\infty}\left(1-a q^{n}\right), \quad(a, b ; q)_{\infty}=(a ; q)_{\infty}(b ; q)_{\infty} .
$$

These identities were first proved by Rogers in 1894 [13], Ramanujan and Rogers in 1919 [14], and independently by Schur in 1917 [15]. In particular, Schur gave an ingenious proof that relied on the integer partition interpretation and used a clever sign-reversing 
involution on pairs of partitions to establish the identities. Throughout the last century, many proofs and generalizations have been given in the literature. For a survey of proofs before 1989, see [1].

In [5], we gave a generalization of the classical Rogers-Ramanujan identities, writing the infinite sum as a linear combination of the infinite products in (1) and (2).

Theorem 1.1. For $m \geq 0$, an integer,

$$
\sum_{n=0}^{\infty} \frac{q^{n^{2}+m n}}{(q ; q)_{n}}=\frac{(-1)^{m} q^{-\left(\begin{array}{c}
m \\
2
\end{array}\right)} c_{m}(q)}{\left(q, q^{4} ; q^{5}\right)_{\infty}}-\frac{(-1)^{m} q^{-\left(\begin{array}{c}
m \\
2
\end{array}\right)} d_{m}(q)}{\left(q^{2}, q^{3} ; q^{5}\right)_{\infty}}
$$

where

$$
\begin{aligned}
& c_{m}(q)=\sum_{\lambda}(-1)^{\lambda} q^{\lambda(5 \lambda-3) / 2}\left[\begin{array}{c}
m-1 \\
\left\lfloor\frac{m+1-5 \lambda}{2}\right\rfloor
\end{array}\right]_{q}, \\
& d_{m}(q)=\sum_{\lambda}(-1)^{\lambda} q^{\lambda(5 \lambda+1) / 2}\left[\begin{array}{c}
m-1 \\
\left\lfloor\frac{m-1-5 \lambda}{2}\right\rfloor
\end{array}\right]_{q} .
\end{aligned}
$$

As usual, $\lfloor x\rfloor$ denotes the greatest integer function and the $q$-binomial coefficients are defined as follows:

$$
\left[\begin{array}{c}
n+m \\
n
\end{array}\right]_{q}= \begin{cases}\frac{\left(q^{n+1} ; q\right)_{m}}{(q ; q)_{m}}, & \text { if } m \geq 0 \text { is an integer } \\
0, & \text { otherwise. }\end{cases}
$$

It is customary to omit the subscript in the case where it is $q$. In future use we will only include the subscript if it differs from $q$.

In a similar spirit as Theorem 1.1, Andrews, Knopfmacher and Knopfmacher proved the following polynomial identity that implies (3) and leads to a simple combinatorial proof. (See [2]) Their motivation was to prove (3) via their method of Engel Expansion.

Theorem 1.2. For integers $m \geq 0$ and $k \geq 1$,

$$
c_{m}(q) d_{m+k}(q)-c_{m+k}(q) d_{m}(q)=(-1)^{m} q^{\left(\begin{array}{c}
m \\
2
\end{array}\right)} \sum_{j \geq 0}\left[\begin{array}{c}
k-1-j \\
j
\end{array}\right] q^{j^{2}+m j},
$$

where the $c_{m}(q)$, and $d_{m}(q)$ are defined as in Theorem 1.1 .

To see how (3) follows from this polynomial identity we must first recall Jacobi's triple product identity:

$$
\sum_{k=-\infty}^{\infty} q^{k^{2}} z^{k}=\left(q^{2},-q z,-q / z ; q^{2}\right)_{\infty}
$$

Letting $k \rightarrow \infty$ in (7) and applying the Jacobi Triple Product identity, one gets (3). Andrews et al. proved Theorem 1.2 by showing that both sides satified the same recurrence, and in particular, that the $c_{m}(q)$ 's, and $d_{m}(q)$ 's satisfy the recurrence relation $f_{m+2}=f_{m+1}+q^{m} f_{m}$ with initial conditions $c_{0}=d_{1}=1$ and $c_{1}=d_{0}=0$. 
In this paper we will prove a determinant identity that specializes to Theorem 1.2 and also works in great generality. The main theorem, Theorem 2.2, is in Section 2. We will use weighted lattice paths to give a combintorial proof of the determinant identity which generalizes a known orthogonal polynomial lemma that implies Theorem 1.1. Section 3 contains some known and some new applications of the $2 \times 2$ determinant identity. In Section 4 , we will give an analagous lattice path proof of a polynomial identity related to our main theorem and state some new generalizations of known Rogers-Ramanujan type identities.

\section{Combinatorics of Associated Polynomials}

In order to understand Theorem 1.2 from a combinatorial perspective, we need to focus on the polynomials in question. As the polynomials satisfy a three-term recurrence, it is natural to look to the theory of orthogonal polynomials for some insight. In [7], Ismail et al. showed there exists an orthogonal polynomial lemma which proves Theorem 1.2. We will first review some well known facts about orthogonal polynomials in order to generalize the result discussed in [7].

Any sequence of orthogonal polynomials, $\left\{p_{n}(x)\right\}$, satisfies a three-term recurrence relation

$$
p_{n+1}(x)=\left(a_{1}(n) x+a_{2}(n)\right) p_{n}(x)+a_{3}(n) p_{n-1}(x), \quad n \geq 1,
$$

where we assume the initial conditions

$$
p_{0}(x)=1, \quad p_{1}(x)=a_{1}(0) x+a_{2}(0)
$$

and the $a_{1}(n), a_{2}(n)$ and $a_{3}(n)$ are sequences of constants with respect to $x$.

The polynomials $\left\{p_{n}^{*}(x)\right\}$ associated with $\left\{p_{n}(x)\right\}$ are defined to be the solutions of

$$
p_{n+1}(x)=\left(a_{1}(n) x+a_{2}(n)\right) p_{n}(x)+a_{3}(n) p_{n-1}(x), \quad n \geq 1,
$$

with the initial conditions,

$$
p_{0}^{*}(x)=0, \quad p_{1}^{*}=a_{1}(0) .
$$

These two sets of polynomials form a basis for the solution set of the three-term recurrence in (9). We can also consider $\left\{p_{n}^{*}(x)\right\}$ as solutions of the three-term recurrence relation with the indices shifted up by one. Therefore, by shifting the indices by more than one, there exist natural generalizations of the idea of associated polynomials. See [12] for a discussion of the associated classical orthogonal polynomials. We may define the $m$ th associated polynomials to be the solutions of:

$$
p_{n+1}^{(m)}(x)=\left(a_{1}(n+m) x+a_{2}(n+m)\right) p_{n}^{(m)}(x)+a_{3}(n+m) p_{n-1}^{(m)}(x), \quad n \geq 1,
$$

with

$$
p_{0}^{(m)}(x)=1, \quad p_{1}^{(m)}=a_{1}(m) x+a_{2}(m)
$$


The combinatorics of general orthogonal polynomials are well understood in terms of lattice paths. (See [19] for details.) In short, in light of the three-term recurrence, one can interpret a specific polynomial $p_{n}(x)$ as a sum over certain weighted paths. Let $E_{n}^{1}$ be the set of paths of length $n$ starting at $(1,0)$ with three types of weighted edges, NN, NE, and N. Let $E_{n}^{2}$ be the set of paths of length $n-1$ starting at $(2,1)$ with the same types of edges. A NN edge which starts at $(i, j)$ and ends at $(i, j+2)$ has weight $a_{3}(j+1)$. A NE edge which starts at $(i, j)$ and ends at $(i+1, j+1)$ has weight $a_{1}(j) x$. Finally, a $\mathrm{N}$ edge which starts at $(i, j)$ and ends at $(i, j+1)$ has weight $a_{2}(j)$. The weight of a path $\lambda$, which we denote by $w t(\lambda)$, is defined to be the product of the weights of the edges in the path.

Now we can write the polynomials that satisfy the three-term recurrence, (9), as a sum over these lattice paths.

$$
\begin{aligned}
& p_{n}(x)=\sum_{\lambda \in E_{n}^{1}} w t(\lambda) \\
& p_{n}^{*}(x)=a_{1}(0) \cdot \sum_{\lambda \in E_{n}^{2}} w t(\lambda) .
\end{aligned}
$$

(Note: Because of the choice of initial conditions for $p_{n}^{*}(x)$, we need to include the constant $a_{1}(0)$ in the lattice path definition.)

Ismail, Prodinger and Stanton were first to show that polynomial identities of the type proved by Andrews et al. were simply special cases of Lemma 2.1, [7]. In this section, we will use lattice paths to give a combinatorial proof of this lemma. It should be noted that such determinants of linearly independent solutions of difference equations are simply discrete analogues of the Wronskian called Casorati determinants. See [10] for background on the Casorati determinant.

The lattice path proof we will give also neatly generalizes to give a determinant identity that will be used to prove various Rogers-Ramanujan type identities. This technique is not new, it is orginally due to Lindström [11]. Related determinantal identites have been studied by Slater, Karlin and McGregor and Gesseal and Viennot [17], [9] and [6]. Gessel and Viennot have used similar techniques to solve several interesting combinatorial problems.

Lemma 2.1. The associated polynomials $p_{n}^{(m)}(x)$ satisfy

$$
p_{n}^{(m)}(x)=\frac{p_{m-1}^{*}(x) p_{n+m}(x)-p_{m-1}(x) p_{n+m}^{*}(x)}{(-1)^{m} a_{3}(1) a_{3}(2) \cdots a_{3}(m-1) a_{1}(0)} .
$$

Proof. First consider the $2 \times 2$ determinant

$$
D_{n, m}=\left|\begin{array}{ll}
p_{n+m}(x) & p_{m-1}(x) \\
p_{n+m}^{*}(x) & p_{m-1}^{*}(x)
\end{array}\right| .
$$

Since the $p(x)$ 's and $p^{*}(x)$ 's are sums over sets of lattice paths, it is easy to see that $D_{n, m}$ can be thought of as a difference of pairs of paths, where we define the weight of a pair of paths as the product of the weights of the individual paths. 
Notice $D_{n, m}=p_{m-1}^{*}(x) p_{n+m}(x)-p_{m-1}(x) p_{n+m}^{*}(x)$ can be interpreted as

$$
D_{n, m}=\sum_{(\lambda, \mu) \in\left(E_{n+m}^{1} \times E_{m-1}^{2}\right)} w t(\lambda) w t(\mu)-\sum_{\left(\lambda^{\prime}, \mu^{\prime}\right) \in\left(E_{n+m}^{2} \times E_{m-1}^{1}\right)} w t\left(\lambda^{\prime}\right) w t\left(\mu^{\prime}\right) .
$$

Consider pairs of paths $(\lambda, \mu)$, where $\lambda$ begins at $(1,0)$ and $\mu$ begins at $(2,1)$. We will define a weight-preserving involution, $\phi$, on pairs of paths. Given a pair of paths, $(\lambda, \mu)$, $\phi((\lambda, \mu))$ is obtained by finding the smallest $y$-coordinate where both paths start a new edge then swapping the edges above that node.

The idea of the involution is shown in the example below in Figures 1 and 2. In Figure 1 , the smallest $y$-coordinate where both paths have a node in common is $y=5$. Figure 2 shows the result of "swapping the tails" of the paths.

Clearly the weights are preserved, as weights depend only on edges and the "swapping" process neither deletes nor adds edges to the pair of paths. We need to check that a pair of paths $(\lambda, \mu) \in E_{n+m}^{1} \times E_{m-1}^{2}$ is mapped to a pair $\left(\lambda^{\prime}, \mu^{\prime}\right) \in E_{n+m}^{2} \times E_{m-1}^{1}$ and vice versa. But this is trivial. If $(\lambda, \mu)$ is in $E_{n+m}^{1} \times E_{m-1}^{2}$, then $\lambda$ begins at $(1,0)$ and has length $n+m$, while $\mu$ begins at $(2,1)$ and has length $m-2$. If we apply the involution, $\phi((\lambda, \mu))=\left(\lambda^{\prime}, \mu^{\prime}\right)$ where the length of $\lambda^{\prime}$ is $m-1$ and the length of $\mu^{\prime}$ is $n+m-1$. We clearly end up in the set $E_{n+m}^{2} \times E_{m-1}^{1}$. Thus, $\phi$ is the desired involution. Now, considering $D_{n, m}$, all pairs of paths for which the involution is valid will cancel. We are left to find the fixed points of the involution.

Assume $m$ is even. The fixed points are pairs of paths where no two edges begin at the same $y$-coordinate. This can only happen if the shorter path contians only NN edges and the longer path is made up of NN edges followed by arbitrary edges for $y$-coordinates greater than the largest $y$-coordinate of the shorter path. In the case $m$ is even, the fixed points are pairs of paths $(\lambda, \mu)$ where $\lambda$ begins at $(1,0)$, has length $m+n$, and the first $m / 2$ edges are of type NN. In addition, $\mu$ begins at $(2,1)$, has length $m-2$, and is made up entirely of NN edges. Since a NN edge from $(i, j)$ to $(i, j+2)$ has weight $a_{3}(j)$, the $\mathrm{NN}$ edges in this set of fixed points contribute the weight $a_{3}(1) a_{3}(2) \cdots a_{3}(m-1) a_{1}(0)$. The edges of $\lambda$ which remain begin at $y=m$ and end at $y=m+n$, but this is simply the shifted polynomial $p_{n}^{(m)}(x)$. This proves

$$
D_{n, m}=a_{3}(1) a_{3}(2) \cdots a_{3}(m) a_{1}(0) p_{n}^{(m)}(x) .
$$

A similar calculation for $m$ odd can be done to complete the proof of the lemma.

As we wish to generalize the notion of associated polynomials, we should note that the polynomials, $p_{n}(x)$ and $p_{n}^{*}(x)$, which satisfy (9) can be written as special cases of the $m$-th associated polynomials defined in (13). In particular

$$
p_{n}(x)=p_{n}^{(0)}(x) \quad \text { and } \quad p_{n}^{*}(x)=a_{1}(0) p_{n-1}^{(1)}(x) \quad \text { for all } n .
$$

A generalization of Lemma 2.1 can be found by defining polynomials with a recurrence of aribtrary length. Although these polynomials are not orthogonal, we will be able to apply a similar lattice path theory. It should be noted that recent developments in the area 


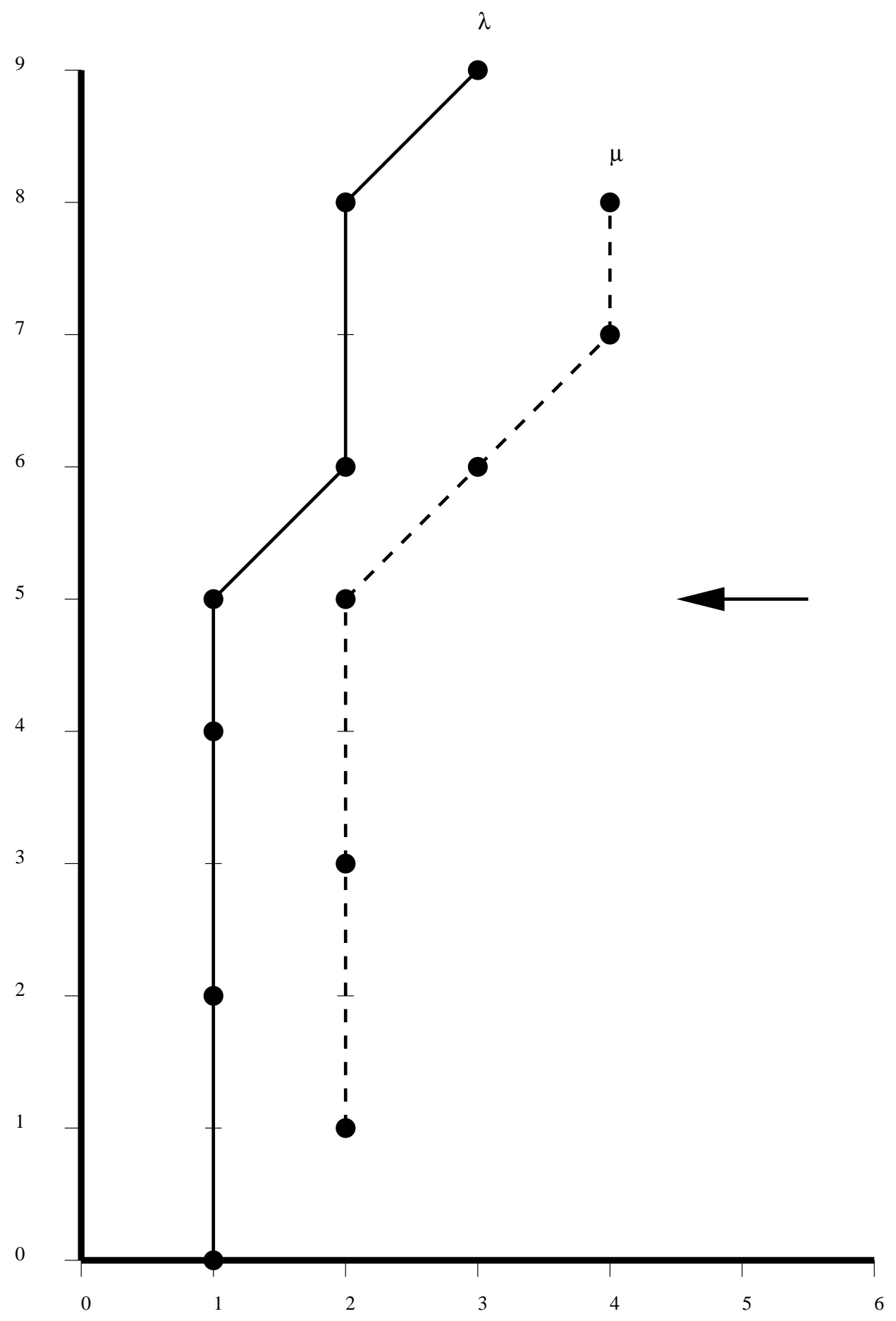

Figure 1: The weights of $\lambda$ and $\mu$ are given by: $w t(\lambda)=a_{3}(1) a_{3}(2) a_{2}(4) a_{1}(5) a_{3}(7) a_{1}(8) x^{2}$ and $w t(\mu)=a_{3}(2) a_{3}(4) a_{1}(5) a_{1}(6) a_{2}(7) x^{2}$. 


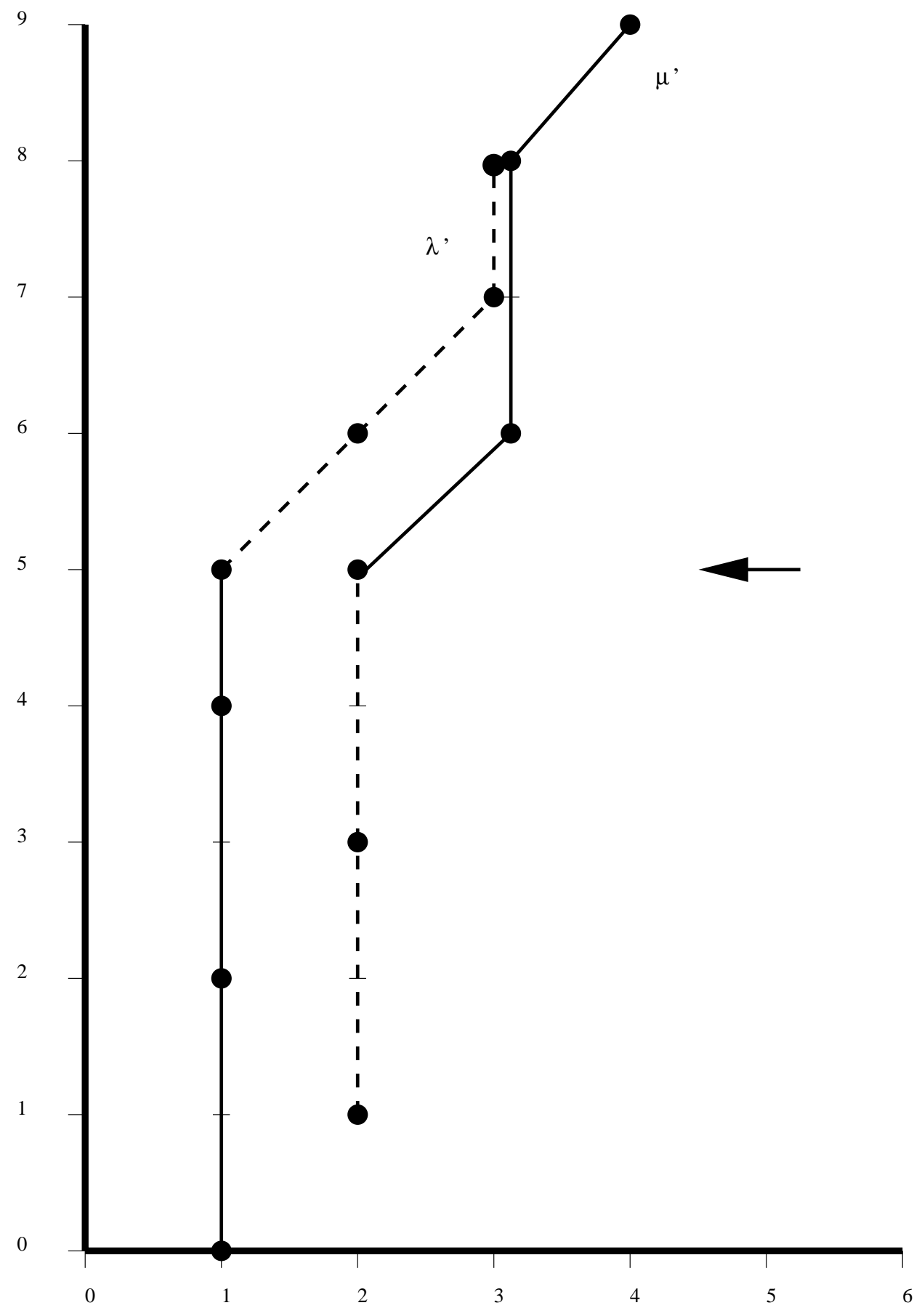

Figure 2: The weights of $\lambda^{\prime}$ and $\mu^{\prime}$ are given by: $w t\left(\lambda^{\prime}\right)=a_{3}(1) a_{3}(2) a_{2}(4) a_{1}(5) a_{1}(6) a_{2}(7) x^{2}$ and $w t\left(\mu^{\prime}\right)=a_{3}(2) a_{3}(4) a_{1}(5) a_{3}(7) a_{1}(8) x^{2}$. 
of multiple orthogonal polynomials have given rise to simialr polynomials with recurrences of arbitrary length. The proof of this theorem is a direct generalization of the lattice path proof given above. Given a recurrence,

$$
p_{n+1, d}^{(m)}(x)=\left(a_{1}(n+m) x+a_{2}(n+m)\right) p_{n, d}^{(m)}(x)+\sum_{j=1}^{d-1} a_{j+2}(n+m) p_{n-j, d}^{(m)}(x),
$$

define general associated polynomials $p_{n, d}^{(m)}(x)$ which satisfy $(20)$ with the following initial conditions:

$$
\begin{aligned}
& p_{0, d}^{(m)}(x)=1 \\
& p_{1, d}^{(m)}(x)=a_{1}(m) x+a_{2}(m), \\
& p_{n, d}^{(m)}(x)=0 \quad \text { if } \quad(1-d)<n<0 .
\end{aligned}
$$

We now state the main theorem.

Theorem 2.2. Let $d, c_{1}, c_{2}, \cdots c_{d-1}$ be positive integers and let $\gamma$ be an integer. Then

$$
\begin{array}{|c}
\left|\begin{array}{cccc}
p_{n, d}^{(\gamma)}(x) & p_{n+c_{1}, d}^{(\gamma)}(x) & \cdots & p_{n+c_{d-1}, d}^{(\gamma)}(x) \\
p_{n-1, d}^{(\gamma+1)}(x) & p_{n-1+c_{1}, d}^{(\gamma+1)}(x) & \cdots & p_{n-1+c_{d-1}, d}^{(\gamma+1)}(x) \\
\vdots & & \ddots & \vdots \\
p_{n-d+1, d}^{(\gamma+d-1)}(x) & p_{n-d+1+c_{1}, d}^{(\gamma+d-1)}(x) & \cdots & p_{n-d+1+c_{d-1}, d}^{(\gamma+d-1)}(x)
\end{array}\right|=(-1)^{n(d-1)} \prod_{i=2}^{n+1} a_{d+1}(i+\gamma) \\
\left|\begin{array}{ccccc}
p_{c_{1}-1, d}^{(\gamma+n+1)}(x) & p_{c_{1}-2, d}^{(\gamma+n+2)}(x) & \cdots & p_{c_{1}-(d-1), d}^{(\gamma+n+d-1)}(x) \\
p_{c_{2}-1, d}^{(\gamma+n+1)}(x) & p_{c_{2}-2, d}^{(\gamma+n+2)}(x) & \cdots & p_{c_{2}-(d-1), d}^{(\gamma+n+d-1)}(x) \\
\vdots & & \ddots & \vdots \\
p_{c_{d-1}-1, d}^{(\gamma+n+1)}(x) & p_{c_{d-1}-2, d}^{(\gamma+n+2)}(x) & \cdots & p_{c_{d-1}-(d-1), d}^{(\gamma+n+d-1)}(x)
\end{array}\right|
\end{array}
$$

Proof. We will proceed by describing the combinatorics of the generalized associated polynomials and describing an involution on $d$-tuples of lattice paths in the same way we proved Lemma 2.1.

We will define lattice paths as we did in the $2 \times 2$ case. Given the $(d+1)$-term recurrence, we consider $(d+1)$ types of edges, $\mathrm{N}, \mathrm{NE}$, and $\mathrm{N}^{(k)}$, where $2 \leq k \leq(d+1)$ and $\mathrm{N}^{(k)}$ is an edge of length $k$. We define the weights of the edges as we did in the previous case. A NE edge from $(i, j)$ to $(i+1, j+1)$ has weight $a_{1}(j) x$, a $\mathrm{N}$ edge beginning at $(i, j)$ and ending at $(i, j+1)$ has weight $a_{2}(j)$, and a $\mathrm{N}^{(k)}$ edge beginning at $(i, j)$ and ending at $(i, j+k)$ has weight $a_{k+1}(j+k-1)$. Again, define the weight of a path made up of these types of edges as the product of the weights of the individual edges.

Given an integer $\gamma$, let $E_{n}^{i+\gamma}$, for $0 \leq i \leq d-1$, be the set of lattice paths of length $n-i$ beginning at $(i+1, i+\gamma)$ made up of the previously defined weighted edges. Then, as in the previous case, the recurrence and initial conditions imply that we can define the polynomials, $p_{n-i, d}^{(i+\gamma)}(x)$, as sums over lattice paths. 


$$
p_{n-i, d}^{(i+\gamma)}(x)=\sum_{\lambda_{i} \in E_{n}^{i+\gamma}} w t\left(\lambda_{i}\right)
$$

The left hand side of Theorem 2.2, which we will call $D$, can now be written in terms of the lattice paths. For simpler notation, we will define $c_{0}=0$.

$$
\begin{aligned}
D & =\sum_{\sigma \in S_{d}}(-1)^{\operatorname{sign}(\sigma)} p_{n+c_{\sigma(0)}, d}^{(\gamma)} p_{n-1+c_{\sigma(1)}, d}^{(\gamma+1)} \cdots p_{n-d+1+c_{\sigma(d-1)}, d}^{(\gamma+d-1)} \\
& =\sum_{\sigma \in S_{d}}(-1)^{\operatorname{sign}(\sigma)} \sum_{\substack{\left(\lambda_{0}, \lambda_{1}, \ldots, \lambda_{d-1}\right) \\
\lambda_{i} \in E_{n+c_{\sigma(i)}}^{i+\gamma}}} w t\left(\lambda_{0} \lambda_{1} \cdots \lambda_{d-1}\right)
\end{aligned}
$$

We now define the involution $\phi$ on $d$-tuples of paths. Let $\left(\lambda_{0}, \lambda_{1}, \ldots, \lambda_{d-1}\right) \in E_{n+c_{\sigma(0)}}^{\gamma} \times$ $E_{n+c_{\sigma(1)}}^{\gamma+1} \times \cdots \times E_{n+c_{\sigma(d-1)}}^{\gamma+d-1}$. Find the smallest $y$-coordinate where all $d$ of the paths have an edge and at least two paths begin a new edge. Swap the tails of those paths. If there are more than two paths beginning a new edge, swap the tails of the two paths with smallest indices. This is clearly an involution. We need to check that the sign is reversed, the weights are preserved, and count the fixed points.

The application of the involution $\phi$ is essentially the multiplication of an element of the symmetric group by a transposition resulting in a change of sign of the permutation. Also, as we are only moving edges, the weight of a product of paths (depending solely on the weight of edges) is preserved. It remains to find the fixed points.

We will assume without loss of generality, that $c_{0}<c_{1}<c_{2}<\cdots<c_{d-1}$. It is possible to do this because if any two of the $c_{i}$ were equal, Theorem 2.2 would be trivially true as both sides would be equal to 0 .

It is apparent that we may apply the involution unless we cannot find a $y$-coordinate where all paths have an edge and where at least two paths begin a new edge. Not being able to find such a $y$-coordinate will only occur if the paths are made up of initial segments of $\mathrm{N}^{(d)}$ edges and the shortest path is composed of $\mathrm{N}^{(d)}$ edges. Let us assume that $n$ is multiple of $d$. Then the fixed points are all $d$-tuples of paths $\left(\lambda_{0}, \lambda_{1}, \ldots, \lambda_{d-1}\right)$ where $\lambda_{0}$ is made up entirely of $\mathrm{N}^{(d)}$ edges, and the remaining $\lambda_{i}$ have the first $n / d$ edges of type $\mathrm{N}^{(d)}$ and the remaining edges are arbitrary. This simply leaves us with the products of weights of the $\mathrm{N}^{(d)}$ edges times $(d-1)$-tuples of paths which have been shifted up by $n$. Summing over all possible $(d-1)$-tuples of paths gives the right hand side of Theorem 2.2. We leave the remaining details, the cases that $n$ is not a mulitple of $d$, to the reader.

\section{Applications}

In this section we give two applications of Lemma 2.1. The first is the polynomial version of Rogers-Ramanujan of Andrews et. al. The second comes from an example in Slater [18] and is new. Recall Thereom 1.2. 
Lemma 3.1. For integers $m \geq 0$ and $k \geq 1$,

$$
c_{m}(q) d_{m+k}(q)-c_{m+k}(q) d_{m}(q)=(-1)^{m} q^{\left(\begin{array}{c}
m \\
2
\end{array}\right)} \sum_{j \geq 0}\left[\begin{array}{c}
k-1-j \\
j
\end{array}\right] q^{j^{2}+m j},
$$

where the $c_{m}(q)$ 's, and $d_{m}(q)$ 's satisfy the recurrence relation $f_{m+2}=f_{m+1}+q^{m} f_{m}$ with initial conditions $c_{1}=0, c_{2}=1, d_{1}=1$, and $d_{2}=1$.

Proof. Our proof is a direct result of Lemma 2.1 Set $a_{1}(n)=1$ for all $n, a_{2}(n)=0$ for all $n$, and $a_{3}(n)=q^{n}$ and let $x=1$ in Lemma 2.1. We then have $d_{m+1}=p_{m}(1)$ and $c_{m+1}=p_{m}^{*}(1)$, so Lemma 2.1 gives

$$
p_{n}^{(m)}(1)=\frac{c_{m}(q) d_{n+m+1}(q)-d_{m}(q) c_{n+m+1}(q)}{(-1)^{m} q^{\left(\begin{array}{c}
m \\
2
\end{array}\right)}} .
$$

If we set $n=k-1$, we find

$$
p_{k-1}^{(m)}(1)=\frac{c_{m}(q) d_{k+m}(q)-d_{m}(q) c_{k+m}(q)}{(-1)^{m} q^{\left(\begin{array}{c}
m \\
2
\end{array}\right)}} .
$$

It remains to evaluate the polynomials $p_{k-1}^{(m)}(1)$. In light of our path argument, $p_{k-1}^{(m)}(1)$ is the generating function for lattice paths of length $k-1$ that begin at $y=m$. This generating function can be found by solving the recurrence satisfied by these associated polynomials in (17) given the initial conditions, $p_{0}^{(m)}(x)=1$ and $p_{1}^{(m)}(x)=a_{1}(m) x+a_{2}(m)$. It is easily seen that this solution gives the right hand side of Lemma 3.1.

Lemma 3.1 is considered a polynomial version of Rogers-Ramanujan because by letting $k \rightarrow \infty$ and using (1) to evaluate the resulting products, we obtain Theorem 1.1.

We will now give another example with a different three term recurrence, $G_{n+1}=$ $-q^{2(n)-1} x G_{n}+G_{n-1}$, that will produce a new generalization of one of the identities from Slater's list. [18].

Theorem 3.2. For $n$ and $m$ positive integers,

$$
(-1)^{m} g_{n}^{(m+2)}(1)=g_{m}^{(1)}(1) g_{n+m}^{(2)}(1)-g_{m-1}^{(2)}(1) g_{n+m+1}^{(1)}(1),
$$

where

$$
g_{n}^{(m)}(1)=\sum_{j=0}^{\lfloor n / 2\rfloor}\left[\begin{array}{c}
n-j \\
j
\end{array}\right]_{q^{4}}(-1)^{n} q^{(2 m-2)(n-2 j)+(n-2 j)^{2}} .
$$

Proof. Consider the recurrence $G_{n+1}=-q^{2(n)-1} x G_{n}+G_{n-1}$. It is simple to solve the recurrence with the shifted coefficients to obtain the closed form for $g_{n}^{(m)}(q)$. As we wish to interpret this as the sum over paths of length $n$ beginning at $y=m$ with NN edges weighted by 1 and NE edges beginning at $y=n$ weighted by $-q^{2(n+m)-1}$, we use the initial conditions $g_{0}^{(m)}(1)=1$ and $g_{1}^{(m)}(1)=-q^{2 m-1}$. We then consider pairs of paths 
beginning at $y=1$ and $y=2$ of lengths $m$ and $n+m$ respectively and perform the same involution we used to prove Lemma 2.1.

Remark: Theorem 3.2 can be proven directly from Lemma 2.1 but the details are slightly more complicated so we appeal to the lattice path interpretation above to simplify the proof.

It can be shown, upon letting $n \rightarrow \infty$ in the above theorem, that Theorem 3.2 gives a $m$-generalization of Slater's identities (SL98) and (SL99) where $q$ has been replaced by $q^{4}$.

Corollary 3.3. For $m$ a positive even integer,

$$
\begin{aligned}
\sum_{j=0}^{\infty} \frac{q^{4 j^{2}+4(m+1) j}}{\left(q^{4} ; q^{4}\right)_{2 j}} & =g_{m}^{(1)}(1) \sum_{j=0}^{\infty} \frac{q^{4 j^{2}+4 j}}{\left(q^{4} ; q^{4}\right)_{2 j}}+g_{m-1}^{(2)}(1) \sum_{j=0}^{\infty} \frac{q^{4 j^{2}+4 j+1}}{\left(q^{4} ; q^{4}\right)_{2 j+1}} \\
& =g_{m}^{(1)}(1) \frac{\left(q^{4}, q^{36}, q^{40} ; q^{40}\right)_{\infty}\left(q^{32}, q^{48} ; q^{80}\right)_{\infty}}{\left(q^{4} ; q^{4}\right)_{\infty}} \\
& +q g_{m-1}^{(2)}(1) \frac{\left(q^{12}, q^{28}, q^{40} ; q^{40}\right)_{\infty}\left(q^{16}, q^{64} ; q^{80}\right)_{\infty}}{\left(q^{4} ; q^{4}\right)_{\infty}}
\end{aligned}
$$

Proof. In order to take the limit as $n \rightarrow \infty$ in Theorem 3.2 we need to consider the closed forms for the polynomials. We will first multiply through Equation (29) by $(-1)^{n+m}$. Assume $n$ and $m$ are even, send $j$ to $n / 2-j$ in the closed form for $g_{n}^{(m+2)}(1)$, and send $j$ to $(n+m) / 2-j$ in the closed forms for both $g_{n+m}^{(2)}(1)$ and $g_{n+m+1}^{(1)}(1)$ to remove the $n$ dependence on powers of $q$ in the polynomials. We can then let $n \rightarrow \infty$ to obtain (30). We can now use Slater's list to evaluate the sums on the right hand side in terms of products.

We will state the $m$ odd case for completeness.

Corollary 3.4. For $m$ a positive odd integer,

$$
\begin{aligned}
\sum_{j=0}^{\infty} \frac{q^{4 j^{2}+4(m+1) j}}{\left(q^{4} ; q^{4}\right)_{2 j}} & =g_{m}^{(1)}(1) \sum_{j=1}^{\infty} \frac{q^{4 j^{2}-1}}{\left(q^{4} ; q^{4}\right)_{2 j-1}}+g_{m-1}^{(2)}(1) \sum_{j=0}^{\infty} \frac{q^{4 j^{2}}}{\left(q^{4} ; q^{4}\right)_{2 j}} \\
& =g_{m}^{(1)}(1) \frac{\left(q^{12}, q^{28}, q^{40} ; q^{40}\right)_{\infty}\left(q^{16}, q^{64} ; q^{80}\right)_{\infty}}{\left(q^{4} ; q^{4}\right)_{\infty}} \\
& +g_{m-1}^{(2)}(1) \frac{\left(q^{8}, q^{32}, q^{40} ; q^{40}\right)_{\infty}\left(q^{24}, q^{56} ; q^{80}\right)_{\infty}}{\left(q^{4} ; q^{4}\right)_{\infty}}
\end{aligned}
$$

We can appeal to Theorem 2.2 to prove many more Rogers-Ramanujan type identities by considering different three-term recurrences. For recurrence of order higher than three, it should be noted that Theorem 2.2 can be iterated by choosing appropriate values for the parameter $\gamma$. However, we can more easily address recurrences of higher order by stating a related theorem. 


\section{Negative (m)-version of Rogers-Ramanujan}

We showed in Section 2 that the Andrews et al. finite $m$-generalization of the classical Rogers-Ramanujan identity, (7), can be proved by interpreting the polynomials in terms of lattice paths and applying an involution. In this section we will note that a simple extension of the lattice path idea gives a bijection which proves the following related result of Carlitz [3]:

Theorem 4.1. For $m \geq 0$,

$$
\sum_{n=0}^{\infty} \frac{q^{n^{2}-m n}}{(q ; q)_{n}}=\frac{v_{m}(q)}{\left(q, q^{4} ; q^{5}\right)_{\infty}}+\frac{u_{m}(q)}{\left(q^{2}, q^{3} ; q^{5}\right)_{\infty}}
$$

where $u_{m}(q)$ and $v_{m}(q)$ both satisfy the same three term recurrence relation,

$$
f_{m+1}=f_{m}+q^{-m} f_{m-1} .
$$

The initial conditions are $u_{0}(q)=0$ and $u_{1}(q)=v_{0}(q)=v_{1}(q)=1$.

Remark: It is easy to check that the Laurent polynomials, $u_{m}(q)$ and $v_{m}(q)$, relate to the polynomials for the positive $m$-generalization, Theorem 1.1, in the following way: $v_{m}(q)=b_{m+1}(1 / q)$ and $u_{m}(q)=a_{m+1}(1 / q)$.

As Carlitz's theorem is, in essence, a negative $m$ analog of the Rogers-Ramanujan generalization, (3), it would be natural to try to understand the connection between the two theorems. We can see immediately that the $u$ 's and $v$ 's are obtained by simply running the recurrence for the c's and d's in (3) backwards. As we had a lattice path interpretation for the positive $m$-version of Rogers-Ramanujan, we are lead to consider a lattice path interpretation of Carlitz's theorem.

We can naturally extend the definition of the polynomials discussed in Section 2 by looking at paths which begin below the $x$-axis. We recall the definition of the generalized associated polynomials for a three term recurrence:

$$
\begin{aligned}
& p_{k}^{(m)}(x)=\left(a_{1}(k+m-1) x+a_{2}(k+m-1)\right) p_{k-1}^{(m)}(x)+a_{3}(k+m-1) p_{k-2}^{(m)}(x) \\
& p_{0}^{(m)}(x)=1 \\
& p_{1}^{(m)}(x)=a_{1}(m) x+a_{2}(m) .
\end{aligned}
$$

For $m<0$ we can think of these polynomials as "negative analogs" to the $m$ th associated polynomials defined in (21). Their combinatorial interpretation is that of sums over paths of length $k$ which begin at $y=m$ and have the usual weights.

We will now state the negative $m$ analog of the classical orthogonal polynomials lemma, Lemma 2.1.

Theorem 4.2. If $m$ and $k$ are strictly positive integers with $k>m$ and $p_{k}^{(-m)}(x)$ as defined in Equation (34), then

$$
p_{m}^{(-m)}(x) p_{k-m}^{(0)}(x)+a_{3}(0) p_{m-1}^{(-m)}(x) p_{k-m-1}^{(1)}(x)=p_{k}^{(-m)}(x) .
$$


Proof. Consider the left hand side of (35) in terms of disjoint pairs of lattice paths. The $p_{m}^{(-m)}(x) p_{k-m}^{(0)}(x)$ represents a sum over pairs of paths $(\lambda, \mu)$ of the products of weights of $\lambda$ and $\mu$, where $\lambda$ is a path of length $m$ beginning at $y=-m$ and $\mu$ is a path of length $k-m$ beginning at $y=0$. We can instead think of this as a sum over paths of length $k$ which begin at $y=-m$ that do not have a NN edge that crosses the $x$-axis. Similarly, we may think of $a_{3}(0) p_{m-1}^{(-m)}(x) p_{k-m-1}^{(1)}(x)$ as a sum over pairs of paths of length $k$ which begin at $y=-m$ and $d o$ contain a NN edge at $y=0$ which results in the inclusion of the $a_{3}(0)$ term. Clearly this gives us all paths of length $k$ which begin at $y=-m$ with the previously defined weights, but that is simply $p_{k}^{(-m)}(x)$. Thus, we have established Theorem 4.2.

While the combinatorial proof of Theroem 4.2 requires the restrictions on $k$ and $m$, the Theorem is true for all integer values of $k$ and $m$ if we evaluate $p_{-n}^{(m)}(x)$ by running the recurrence (34) backwards.

We note that the following polynomial version of the result of Carlitz is a direct result of Theorem 4.2 .

Corollary 4.3. For $m \geq 1$ and $k \geq m$,

$$
v_{m}(q) b_{k-m}(q)+u_{m}(q) a_{k-m}(q)=\sum_{j=0}^{\left\lfloor\frac{k-1}{2}\right\rfloor}\left[\begin{array}{c}
k-1-j \\
j
\end{array}\right] q^{j^{2}-m j},
$$

where the $u$ 's and $v$ 's are defined above in Theorem 4.1 and the c's and d's are defined in (4).

Clearly, when $k \rightarrow \infty$ in the above identity we obtain Theorem 4.1 , the $(-m)$-version for the classical Rogers-Ramanujan identities.

Similarly to the way the lattice path interpretation of recursively defined polynomials generalizes to a determinant identity for higher order recurrences in Section 2, the above interpretation generalizes as well. We will state the most general theorem and give some examples of new Rogers-Ramanujan type generalizations.

We recall the general associated polynomials $p_{n, d}^{(m)}(x)$ defined in Section 2 . We note that the polynomials are well defined for negative values of $m$. We may now state the theorem which generalizes Theorem 4.2.

Theorem 4.4. For $k>m \geq d-1$ with $k, m$ and $d$ positive integers,

$$
p_{k, d}^{(0)}(x) p_{m, d}^{(-m)}(x)+\sum_{i=2}^{d} \sum_{j=1}^{i-1} a_{i+1}(j-1) p_{k-j, d}^{(j)}(x) p_{m-(i-j), d}^{(-m)}(x)=p_{k+m, d}^{(-m)}(x) .
$$

Proof. We will give a bijection of lattice paths which proves the theorem. The proof is simple once the lattice path interpretation of the shifted polynomials is known. Consider the right hand side of (37). In light of the recurrence satisfied by $p_{k+m, d}^{(-m)}(x)$, we can write

$$
p_{k+m, d}^{(-m)}(x)=\sum_{\lambda \in E_{k+m}^{-m}} w t(\lambda)
$$


where $E_{k+m}^{-m}$ is the set of paths of length $k+m$ which begin at $y=-m$ and are made up of $d+1$ types of edges all defined as in Theorem 2.2.

Now consider the left hand side of (37). We can show that the same weighted paths are counted again, merely split into several subcases. If we choose one of these paths and look at the behavior at $y=0$ there are several possibilities, each one corresponds naturally to a term in the sum on the left side of (37). The path may have a node at $y=0$, in which case it must be a product of two paths, one of length $m$ beginning at $y=-m$ and one of length $k$ beginning at $y=0$. But this is simply the $p_{k, d}^{(0)}(x) p_{m, d}^{(-m)}(x)$ term. Otherwise the path must have an edge of length $i, i \geq 2$, from $y=j-i$ to $y=j$ which obviously crosses the $x$-axis. In this case we get a product of the weight of that particular edge and the two shifted polynomials, $p_{k-j, d}^{(j)}(x)$ and $p_{m-(i-j), d}^{(-m)}(x)$ which give the remaining terms of the left side.

Remark: Note that in the case that $d=2$, Theorem 4.4 implies Theorem 4.2. Like Theroem 4.2, Theorem 4.4 is valid for all integer values of $k$ and $m$ if we define $p_{-k, d}^{(m)}(x)$ by running the recurrence (20) backwards, however the lattice path proof requires the given conditions on $k$ and $m$.

Theorem 4.4 allows us to obtain polynomial identities from recurrences of any order. If we are able to solve the recurrences, we can take limits to obtain $(-m)$-generalizations of Rogers-Ramanujan identities. Although there are several examples of Theorem 4.4 (See [4]), we present one final application here.

We will consider the four term recurrence

$$
P_{n+1}(x)=-q x P_{n}(x)+\left(1+q^{2 n}\right) P_{n-1}(x)+q P_{n-2}(x),
$$

which is motivated by an m-version of Slater's Equations (SL32) and (SL33) found by Ismail and Stanton in [8]. We will apply Theorem 4.4 to the shifted polynomials defined by this recurrence with initial conditions: $p_{0,3}^{(m)}(1)=1, p_{1,3}^{(m)}(1)=-q$, and $p_{n, 3}^{(m)}(1)=0$ for $-3<n<0$ to obtain:

Theorem 4.5. For $n$ and $m$ positive integers with $n>m$,

$$
\begin{aligned}
p_{n+m, 3}^{(-m)}(1) & =p_{m, 3}^{(-m)}(1) p_{n, 3}^{(0)}(1)+2 p_{m-1,3}^{(-m)}(1) p_{n-1,3}^{(1)}(1) \\
& +q p_{m-1,3}^{(-m)}(1) p_{n-2,3}^{(2)}(1)+q p_{m-2,3}^{(-m)}(1) p_{n-1,3}^{(1)}(1)
\end{aligned}
$$

where

$$
p_{n, 3}^{(m)}(1)=\sum_{j=0}^{\left\lfloor\frac{n}{2}\right\rfloor} \sum_{k=0}^{\left\lfloor\frac{n-j}{2}\right\rfloor}\left[\begin{array}{c}
n-2 k-j \\
j
\end{array}\right]_{q^{2}}\left[\begin{array}{c}
j+k \\
k
\end{array}\right]_{q^{4}}(-1)^{n} q^{n+2 j^{2}+2 m j-2 j-2 k} .
$$

Proof. If we consider the four-term recurrence (39), the proof follows directly from Theorem 4.4 with $d=3, a_{1}(n)=-q, a_{2}(n)=0, a_{3}(n)=1+q^{2 n}, a_{4}(n)=q$, and $x=1$. We find the closed form for the polynomials $p_{n, 3}^{(m)}(1)$ by solving the recurrence (39).

We may once again take a limit of the polynomial theorem as $n \rightarrow \infty$ to obtain: 
Corollary 4.6. For $m \geq 0$ an integer, and $p_{n, 3}^{(m)}(x)$ defined to satisfy (39) with the initial conditions discussed above,

$$
\begin{aligned}
(-1)^{m} \sum_{j=0}^{\infty} \frac{q^{2 j^{2}-2(m+1) j+m}}{\left(q^{2} ; q^{2}\right)_{j}\left(q^{2} ; q^{4}\right)_{j}} & =p_{m, 3}^{(-m)}(1) \frac{\left(q^{8}, q^{20}, q^{28} ; q^{28}\right)_{\infty}}{\left(q^{2} ; q^{2}\right)_{\infty}} \\
& -q p_{m-1,3}^{(-m)}(1) \frac{\left(q^{4}, q^{24}, q^{28} ; q^{28}\right)_{\infty}}{\left(q^{2} ; q^{2}\right)_{\infty}} \\
& +q^{-1}\left(p_{m-1,3}^{(-m)}(1)-p_{m+1,3}^{(-m)}(1)\right) \frac{\left(q^{12}, q^{16}, q^{28} ; q^{28}\right)_{\infty}}{\left(q^{2} ; q^{2}\right)_{\infty}} .
\end{aligned}
$$

Proof. Simply let $n \rightarrow \infty$ in Theorem 4.5. The $k$-sum in the closed form for the $p_{n, 3}^{(m)}(1)^{\prime} s$ can be summed and then we use Slater's list, particularly Equations (SL59), (SL60), and (SL61), to evaluate the infinite sums in terms of products. See [18] for details.

Remark: When $m=0$ in Corollary 4.6 we get an identity for the sum of Slater's (SL60) and (SL61). If we let $m=-1$ we need to evaluate $p_{-3,3}^{(0)}(1)$ by running the recurrence backwards. It turns out that $p_{-3,3}^{(0}(1)=q^{-1}$ and we obtain Slater's Equation (SL61) with $q$ replaced by $q^{2}$. Also, if $m=1$ we can get a linear combination of Slater's (SL59), (SL60), and (SL61). Therefore, we have Slater's identity (SL59) as well. Although the proof of Corollary 4.6 requires that $m$ be a positive integer, we do in fact have a valid theorem when $m$ is a negative integer. One can define polynomials $p_{-n, 3}^{(m)}(1)$ by running the recurrrence for the $p_{n, 3}^{(m)}(x)$ 's backwards. If we do this, we find that Corollary 4.6 is valid in the case than $m<0$.

In light of these examples, we can appeal to L.J. Slater's list of 130 Rogers-Ramanujan type identities to investigate which of them may give rise to new theorems. Thirty-nine of her identities can be generalized by inserting a linear power of $q^{m}$ in the numerator to satisfy a homogeneous three term recurrence relation, 59 of them will satisfy a homogeneous four term recurrence relation, and 9 satisfy a homogeneous five term relation. Preliminary investigation indicates that applying Theorem 4.4 in all these cases will lead to a polynomial identity which in many cases seems to be new. Drew Sills has polynomial versions of all of Slater's identities that do not contain the general power of $q^{m},[16]$. Theorem 4.4 allows us to generalize many of his results.

\section{References}

[1] G.E. Andrews, On the proofs of the Rogers-Ramanujan identities in " $q$-Series and partitions", D. Stanton, ed., Springer-Verlag, 1989, 1-14.

[2] G.E. Andrews, A. Knopfmacher, and J. Knopfmacher, Engel Expansions and the Rogers-Ramanujan Identities, J. Number Theory 80 (2000), 273-280.

[3] L. Carlitz, Some formulas related to the Rogers-Ramanujan identities, Annali di Math. (IV) 47 (1959), 243-251. 
[4] K. Garrett, Involutions and Generalizations of Rogers-Ramanujan Type Identities, Ph.D. Thesis, (2001).

[5] K. Garrett, M.E.H. Ismail, and D. Stanton, Variants of the Rogers-Ramanujan Identities, Adv. in Appl. Math. 23 (1999), 274-199.

[6] I. Gessel, G. Viennot, Binomial determinants, paths and hook length formulae, Adv. in Appl. Math. 58 (1985), 300-321.

[7] M.E.H. Ismail, H. Prodinger, and D. Stanton, Schur's determinants and partition theorems, Sem. Loth. Comb. 44 B44a, (2000) 10pp.

[8] M.E.H. Ismail, D. Stanton, Tribasic integrals and identities of Rogers-Ramanujan type, Trans. Amer. MAth. Soc. 355 (2003), 4061-4091.

[9] S. Karlin, J.L McGregor, Coincidence probabilities, Pacific J. Math. 9 (1959), 11411164.

[10] W. G. Kelley, A. C. Peterson, Difference Equations: An Introduction with Applications, Academic Press, San Diego, California, 2001.

[11] B. Lindström, On the vector representation of induced matroids, Bull. London Math. Soc. 5 (1973), 85-90.

[12] M. Rahman, The associated classical orthogonal polynomials in "Special Function 2000: Current Perspective and Future Directions", J. Bustoz, M.E.H. Ismail, S.K. Suslov eds. Proc. NATO ASI, Kluwer, 2001, 255-279.

[13] L.J. Rogers, Second memoir on the expansion of certain infinite products, Proc. London Math. Soc. 25 (1894), 318-343.

[14] L.J. Rogers and S. Ramanujan, Proof of certain identities in combinatory analysis, Proc. Camb. Phil. Soc. 19, 211-216.

[15] I. Schur, Ein Beitrag zur additiven Zahlentheorie und zur Theorie der Kettenbrüche, S.-B Preuss. Akad. Wiss. Phys.-Math. Kl. (1917), 302-321, reprinted in I. Schur, Gesmmelte Abhandungen, volumes 2, 117-136, Springer, Berlin, 1973.

[16] A. Sills, Finite Rogers-Ramanujan Type Identities, Electronic Journal of Combinatorics 10 (2003).

[17] J.C. Slater, The theory of complex spectra, Phys. Rev. 34 (1929), 1293-1322.

[18] L.J. Slater, Further identities of the Rogers-Ramanujan type, Proc. London Math. Soc. (2) 54 (1952), 147-167.

[19] G. Viennot, Une théorie combinatoire des polynômes orthogonaux généraux, Lecture Notes, UQAM, 1983. 